\title{
RETURNS TO TERTIARY EDUCATION IN WESTERN \\ AND EASTERN EUROPE
}

\section{Martina Mysíková, Jiří Večerník*}

\begin{abstract}
In the 1990s, the transition countries in Central and Eastern Europe witnessed an upward trend in returns to education, unlike in Western European countries. This upward trend led to much higher returns than in what was observed in the communist period or compared to the West. The surveys EU-SILC collected since 2005 show that although returns to tertiary education were converging across Europe, there is still a big difference between East and West, with returns considerably higher in the East. Panel analysis reveals also substantial differences in the factors behind returns to tertiary education in the East and the West. The assumed negative relationship between the share of tertiary-educated workers in the working-age population and the returns to tertiary education were confirmed only in the West. The job vacancy rate has a significant negative impact on returns to tertiary education only in the East. While in the West the labour market seems to react more to labour supply, in the East labour demand plays a more important role.
\end{abstract}

Keywords: returns to education, tertiary education, Western and Eastern Europe JEL Classifications: I26, J31, D31, P20

\section{Introduction}

There is a contrast between theory and empirics regarding the trends in private returns to the education of workers. According to mainstream economics, returns are related to the productivity of their work. Therefore, it can be said that when labour productivity rises, and returns to education increase. However, in accordance with the law of diminishing returns to capital, returns to education are expected to decrease with the increasing supply of qualified labour. Accordingly, Becker's "Woytinsky Lecture Hypothesis" (Becker, 1975) was developed in labour economics and claims that marginal returns to education fall as the proportion of the population with higher education rises.

Twenty-five years ago, this explanation was supported by evidence collected over various periods up to the mid-1980s by Psacharopoulos (1989, pp. 227-228) on a set

* Martina Mysíková, Institute of Sociology, Czech Academy of Sciences, Prague, Czech Republic (martina.mysikova@soc.cas.cz);

Jiří Večerník, Institute of Sociology, Czech Academy of Sciences, Prague, Czech Republic (jiri.vecernik@soc.cas.cz).

This work was supported by the Grant No. P402/12/G130. "The Relationships Between Skills, Schooling and Labour Market Outcomes: A Longitudinal Study' from the Czech Grant Agency”. The EU-SILC datasets were made available on the basis of Contract No. 265/14 between the European Commission, Eurostat, and the Institute of Sociology, Czech Academy of Sciences. 
of developed and developing countries. He found that educational expansion is usually followed by a decline in returns to education and concluded that "the evidence seems to be more consistent with the human capital theory of the relationship between education and earnings, rather than the alternative theories of screening, segmentation, or the maintenance of the status quo from generation to generation".

However, returns would also remain stable if the growing supply of better-educated workers was offset by a higher demand for skilled workers as a result of technological change and more complex management in the knowledge-based society. To determine the effect of education on wages, Barth and Lucifora (2006) examined twelve European countries between 1985 and 2000. They found that while the relative supply of tertiary education was on average 20 per cent, such a "supply shock" did not affect the wage premium attached to tertiary education, which remained stable.

In fact, as available evidence suggests, there was no consistent trend in returns to education in West European advanced countries, but their direction and scale varied. In contrast, in the transition countries of Central and Eastern Europe, returns to education increased rapidly and noticeably during market liberalisation. Using the human capital theory to explain their development is insufficient here. Although "communist human capital" was appropriate for capitalist markets (Flanagan, 1998), it cannot be assumed that simply owing to the change in regime labour productivity rose fast enough to explain such an increase in returns.

Fleisher et al. (2005) explanation for the changes is the easing of legal, regulatory, and institutional constraints on wage-setting. Thus, the faster the reforms proceed, the faster the returns to education adjust to market rates. However, the systemic changes went well beyond the economy itself and the rapid increase in returns to education in transition countries is not only a matter of economy. Transition and reforms also brought about a change in the value system, which had important effects on the "market rates" of education. To explain such a change we have to take into account the inferior position of education under the communist value settings and how it changed to the opposite in the transition (Večerník, 2009, Chapter 4).

The aims of this article are: first, to gather available information on changes to returns to education in two past decades; second, to present new facts on recent developments in returns to education in European countries; and, third, to search for explanatory factors of East-West differences.

The second section thus briefly overviews the results that have been collected to date, which in sum indicate that while in Western economies the trends of returns to education differ, in Central European post-communist countries they have increased since 1990. The third section looks at the trends of returns to tertiary education using the EU-SILC surveys of 2005-2014 showing the sustained superiority of transition countries over Western Europe. In the fourth section, a macro-level is added to examine the effect of the increasing supply of educated labour on returns to tertiary education and other factors resulting in a substantial difference between the East and West of Europe. 


\section{Trends in the 1990s: Divergent Paths}

The evidence on the trends in returns to education and their various aspects in the 1990s was based on collecting national data from various sources. In Western European countries, the topic was investigated specifically in two large comparative projects financed by the EU research programmes: the Project "Public Funding and Private Returns to Education" (PURE) described the period 1985-1995 (Harmon et al., 2001), the Project, "Education and Wage Inequality in Europe" (EDWIN) described the period 1984-2003 (Dolton et al., 2009).

The Final Report of the PURE Project stated: "There does not seem to be a clear pattern in the trends, however. In total, there appears to be 15 cases of no trend, 7 cases of increasing returns, and 7 cases of decreasing returns" (PURE, 2001). EDWIN Project concluded: "The countries are divided into four categories: (1) decreasing trend at all educational degree levels with the decline rising with the educational level (France, Portugal, Sweden); (2) opposite trends across educational degree levels with decline only at the tertiary level (Finland, Germany, UK); (3) opposite trends across educational degree levels with increase only at the tertiary level (Norway); (4) increasing trend at all educational degree levels (Greece, Italy)" (EDWIN, 2006).

Among advanced European countries, there are several of them where the returns decrease in line with the theoretical expectation that the more widespread higher skills are, the lower the returns on them compared to lower levels of education. For the UK, Moffitt (2007) estimated marginal returns to higher education and demonstrated that these returns fall as the proportion of the population with higher education rises. For Sweden, a steady decrease of returns to education was documented by Björklund (2000). For Austria, Fersterer and Winter-Ebmer (2003) revealed that returns to education have considerably fallen in the period 1981-1997. For France, Thélot and Selz (2004) stated that returns to education between 1964 and 1985 decreased from 11 per cent in 1964 to 9 per cent in 1985 and have remained stable since.

In contrast to the diverging development in Western countries and the occasional decrease in returns to education, the statistical data for post-communist countries consistently demonstrate increasing returns to education, in particular during the early transition. Extensive literature on this is available on the Czech Republic (Filer et al., 1999; Jurajda, 2003; Munich et al., 2005; Večerník, 2013). The evidence shows a substantial rise also in Hungary, Poland and Slovenia (Kertesi and Köllő, 1999; Rutkowski, 2001; Stanovnik, 1997). The data from those and several other countries (Estonia, Romania, Russia, and Ukraine) were summarized by Fleisher et al. (2005), who showed a fairly steep increase of returns to education in all of them.

\section{Cross Europe Comparison in the 2000s: the Micro-Level}

Psacharopoulos and Patrinos (2004, p. 114) wrote that "the real reason one should be sceptical about indiscriminate rate of return compilations, and in spite of the efforts of the compilers, is that in the original works the estimates are rarely fully comparable. 
There are two main sources of non-comparability: data sample coverage and methodology. Ideally, a rate of return to investment in education should be based on a representative sample of the country's population. But in reality this is the exception rather than the rule". This was valid for the comparison across countries in the 1990s presented above.

However, for the 2000s, two new sources emerged, which allow a better comparison of EU countries. Since 2002, every four years Eurostat produces the cross-national Structure of Earnings Survey (SES), which collects information on earnings in firms and companies with at least 10 employees in the Industry and Services sector. Another source is the survey The European Union Statistics on Income and Living Conditions (EU-SILC), which contains datasets with personal information on gross/net earnings. These data have been available for all EU countries yearly since 2005 .

\subsection{Data sample and variables}

The analysis uses EU-SILC 2005-2014 cross-sectional individual data to estimate returns to tertiary education. The sample includes 29 European countries, of which 11 are new EU member states that have joined in or since 2004 (except for Malta and Cyprus), which for the sake of simplicity are referred to here as "Eastern Europe", and 18 Western European countries (EU-15 plus Switzerland, Iceland and Norway). The income reference period is usually the previous calendar year, except for the UK (current year) and Ireland (the last twelve months). Therefore, returns to education obtained from, for instance, EU-SILC 2005 data are used for the year 2004 and - so far the last available - EU-SILC 2014 data are used for the year 2013.

The selection of prime-age (25-54) respondents ensures that their job choice and corresponding wage level are not influenced either by schooling (in the bottom tail of the age distribution) or by retirement (in the top tail). Current part-time or full-time employees were selected for the wage equation estimation. The self-employed were excluded because their fluctuating earnings would bias the analysis (Heckman, 1979). In order to estimate the first step of the Heckman model, the sample was supplemented with the inclusion of noworking individuals, namely the unemployed and other inactive persons. Students, (early) retired, disabled, and people in compulsory military service were excluded.

The dependent variable in the main wage equation is a logarithm of hourly gross wage. As this variable is not directly available in EU-SILC datasets, it was derived from the gross annual wage adjusted to the number of months of part-time and full-time employment during the year under observation and to the number of hours usually worked per week. In the early years of the EU-SILC survey, countries could choose whether information on net or gross earnings would be surveyed. Data on the gross annual wage were thus missing in several countries in 2005-2006, but, fortunately, information on gross monthly earnings was provided for all these countries except for Latvia. In a majority of countries, hours worked and other job characteristics are related to the current situation, while the earnings are related to the previous calendar year. Therefore, in countries where this variable is available, workers who changed their job during the previous year were excluded in order to avoid the situation where job characteristics and working hours would not correspond to earnings. 
Our primary focus is placed on education, and more precisely on a dummy variable for tertiary education (ISCED 5 and 6). A dummy for low education (ISCED 0 to 2) is included as well. Hence, the reference group consists of respondents with secondary education (ISCED 3 and 4). The wage equation includes a dummy variable for males and work experience measured in years (and its square). The direct variable indicating the number of years spent in paid work is not available, or includes an inacceptable share of missing values in some countries. In these cases, years of work experience were derived as the year of the survey minus the year when the highest level of education was attained.

The wage equation further includes the following set of available job characteristics. First of all, it introduces two dummies for employees working in a local unit with a maximum number of 10 workers and 11-49 workers (reference group 50+ workers). As large companies typically offer higher wages, these two variables are expected to have a negative impact on wage level. We also have a dummy for workers with an open-ended employment contract (reference group covers workers with limited contract, temporary jobs and with jobs without any contract; missing in Denmark and in the UK in several waves), a dummy for a supervisory or managerial position and a dummy for living in a larger city (missing in the Netherlands and Slovenia) and finally 7 dummies for occupation type.

Education and gender dummies are included as explanatory variables in the selection equation. Additionally, this equation includes two dummies for age categories (24-35 and 35-44), dummies for the presence of children aged 0-2 and 3-5 years old, a dummy for living with a partner and a dummy for non-earned income. The latter equals 1 if the unearned household gross income represents more than one-half of the national poverty line, and zero otherwise. Since gross values were missing in the early years of the EU-SILC in several countries, net values were used for Spain, Greece, Italy, Portugal and France.

\subsection{The Heckman selection model methodology}

In our analysis, we use the selection wage model to estimate returns to tertiary education. The Heckman's (1979) selection procedure is a two-stage model. First, a probit model for the probability of having a job is applied. In the second stage, predicted individual probabilities are added as an explanatory variable to the wage equation. If the unobservables in the participation equation are correlated with the unobservables in the wage equation, the estimates without correction (in an OLS model) would be biased. This basically means that the unobservables in the selection (or choice) of working individuals affect also the wage equation. In other words, selection into the sample of working individuals is a nonrandom process affected by different unobservables. The estimated wage function under the selection-corrected Heckman model is:

$$
\ln W_{i}=X_{i} \beta+\rho \sigma_{\varepsilon} \lambda_{i}+\varepsilon_{i}^{*}, \text { where } \lambda_{i}=\frac{\varphi\left(V_{i} y\right)}{\Phi\left(V_{i} y\right)},
$$

where vector $X_{i}$ includes all explanatory variables of the wage equation, $\phi$ and $\Phi$ signify standard normal density and distribution functions, respectively, $V_{i}$ represents the vector of explanatory variables of the participation equation that should differ from 
the one included in the wage equation, $\rho$ is the correlation coefficient of the wage and participation equations and $\sigma_{\varepsilon}$ is the standard deviation. Robust standard errors were applied in order to avoid heteroscedasticity. ${ }^{1}$ The Wald test of the independence equations is used to identify if $\rho$ significantly differs from zero. The results showed that there was no significant selection into employment in several countries in some years. In such cases, however, the coefficient obtained from the Heckman model differed only negligibly from the one obtained from the standard OLS wage regression. The estimations are weighted by personal cross-sectional weights.

\subsection{Results of the analysis}

According to EU-SILC data, the level of returns to tertiary education differs considerably across European countries (Table 1). The coefficients in the Heckman regressions, which indicates that the relative rewards of tertiary-educated workers compared to secondaryeducated ones fluctuate between roughly 0.1 and 0.4 . In the last year of observations, the lowest returns are found in Scandinavian countries and Spain, while the highest returns were identified in Slovenia, Hungary, Portugal, and Latvia. In transition countries in 2013, returns are high overall, but among them, the Czech Republic ranks nearly last (that is the lowest returns) and Romania is the very last.

During the period under observation almost no country displayed a consistent upward or downward trend or a rough stability. On average, the values were slightly increasing up to 2009, after which year they started falling and continued to do so until 2012. The small increase before 2009 on average was caused mainly by the development of returns to tertiary education in transition countries, which was upward, with the exception of Hungary and Poland where the returns were high anyway. In the West, returns to tertiary education increased in some countries as well. The highest increase occurred in Sweden, followed by UK, Spain, and Germany; however, returns to tertiary education remained lower than in any Eastern country in 2009.

The data presented in Table 1 show the year 2009 as a turning point when the up to that time rising returns to tertiary education started to decrease. This has to do with the economic recession at that time. However, the turnaround is not very dramatic and it occurred to a different degree in different countries. In some countries the turning point did not occur until 2010 and in some countries it did not occur at all. Overall, changes over time were much smaller than the differences between countries, which remained on quite a similar level as before. The returns to tertiary education are thus still considerably higher in Eastern than in Western Europe.

We are aware of a possible endogeneity of education. A proper solution would be to apply an instrumental variables regression. However, the instruments for education, i.e. the education of parents, are available from the EU-SILC additional modules on the intergenerational transmission of inequalities fielded only in 2005 and 2011. 


\begin{tabular}{|c|c|c|c|c|c|c|c|c|c|c|c|c|c|c|c|c|c|c|c|c|c|}
\hline 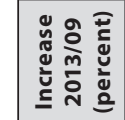 & & $\begin{array}{l}\stackrel{\circ}{0} \\
=\end{array}$ & $\widehat{\infty}$ & 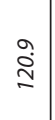 & $\stackrel{Ð}{\underset{\Xi}{ \pm}}$ & @̊ & 亗 & $\underset{\sim}{\mathfrak{d}}$ & 㐫 & $\frac{n}{\infty}$ & $\overline{\tilde{n}}$ & $\begin{array}{l}\infty \\
\stackrel{0}{\infty} \\
\sim\end{array}$ & m & 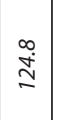 & గై & $\underset{\dot{ \pm}}{=}$ & $\stackrel{m}{\infty}$ & $\overline{\tilde{\theta}}$ & $\begin{array}{l}\stackrel{0}{\alpha} \\
\text { }\end{array}$ & 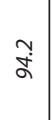 & 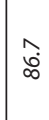 \\
\hline 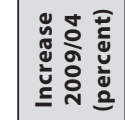 & & $\bar{\infty}$ & $\overline{\check{D}}$ & $\begin{array}{l}0 \\
\text { md } \\
\end{array}$ & $\begin{array}{l}0 \\
\text { îं }\end{array}$ & 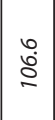 & $\stackrel{\stackrel{0}{m}}{\stackrel{m}{\sim}}$ & $\underset{\infty}{m}$ & $\stackrel{N}{\wedge}$ & 1 & $\stackrel{\stackrel{O}{\grave{I}}}{=}$ & लु & 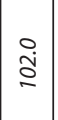 & 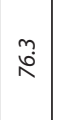 & $\begin{array}{l}\hat{n} \\
\stackrel{0}{=}\end{array}$ & 1 & $\begin{array}{l}n \\
\infty \\
\infty\end{array}$ & $\stackrel{\infty}{\ddot{\sim}}$ & $\stackrel{\hat{\aleph}}{\underline{n}}$ & 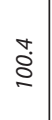 & ஓे \\
\hline 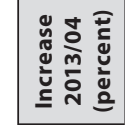 & & $\stackrel{n}{\kappa}$ & ळे & $\underset{\widetilde{N}}{\mathscr{2}}$ & 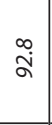 & $\underset{\emptyset}{=}$ & $\begin{array}{l}\text { के } \\
\infty\end{array}$ & 쏫 & $\stackrel{\circ}{\stackrel{\circ}{\prime}}$ & 1 & ڤેं & $\begin{array}{l}\tilde{L} \\
\stackrel{0}{=}\end{array}$ & 華 & బू' & $\stackrel{\sim}{\underset{N}{N}}$ & 1 & $\bar{\AA}$ & 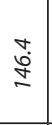 & $\begin{array}{l}\stackrel{0}{\therefore} \\
\text { }\end{array}$ & $\begin{array}{l}0 \\
\dot{d}\end{array}$ & $\begin{array}{l}\mathfrak{n} \\
\stackrel{\infty}{0}\end{array}$ \\
\hline 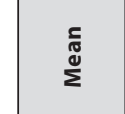 & & $\frac{\bar{n}}{0}$ & 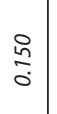 & $\frac{d}{0}$ & $\overline{\grave{o}}$ & $\stackrel{n}{\stackrel{n}{0}}$ & ס̄ & $\begin{array}{l}0 \\
\stackrel{0}{0} \\
0\end{array}$ & $\stackrel{n}{\stackrel{n}{0}}$ & 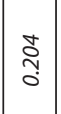 & $\frac{\pi}{0}$ & $\frac{2}{5}$ & $\frac{8}{\frac{8}{0}}$ & $\stackrel{\infty}{\infty}$ & 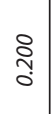 & $\frac{\bar{\sigma}}{0}$ & 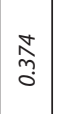 & $\begin{array}{l}\stackrel{0}{0} \\
\stackrel{0}{0}\end{array}$ & $\stackrel{m}{\infty}$ & $\stackrel{\infty}{\stackrel{\infty}{\circ}}$ & oे \\
\hline$\check{m}_{i}^{m}$ & & $\begin{array}{l} \pm \\
\stackrel{0}{0}\end{array}$ & $\frac{\overline{i n}}{\overline{0}}$ & 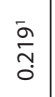 & $\begin{array}{l}\overline{0} \\
\text { ò. } \\
\text { o. }\end{array}$ & $\frac{\infty}{\circ}$ & $\begin{array}{l}\text { mे } \\
\stackrel{m}{0} \\
\text { O. }\end{array}$ & $\begin{array}{l}\bar{\alpha} \\
\hat{0} \\
0\end{array}$ & $\begin{array}{l}\bar{\infty} \\
\stackrel{\infty}{h} \\
\overline{0}\end{array}$ & $\stackrel{\circ}{\circ}$ & $\frac{\bar{n}}{m}$ & $\frac{m}{\sigma}$ & $\begin{array}{l}\overline{\hat{a}} \\
\text { ò }\end{array}$ & $\frac{N}{\stackrel{N}{0}}$ & 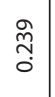 & $\frac{a}{\sigma}$ & 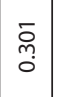 & $\begin{array}{l}\text { ö } \\
\text { Oे. } \\
\text { o. }\end{array}$ & $\begin{array}{c}\infty \\
\infty \\
0\end{array}$ & $\stackrel{\substack{n \\
0}}{\circ}$ & రి \\
\hline$\underset{\sim}{\sim}$ & & $\underset{i}{\stackrel{i}{0}}$ & 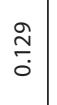 & 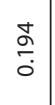 & $\begin{array}{l}\hat{0} \\
0 \\
0\end{array}$ & 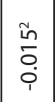 & $\frac{\text { ปี }}{0}$ & $\begin{array}{l}\bar{n} \\
\hat{0} \\
0 \\
0\end{array}$ & $\frac{\bar{j}}{\overline{0}}$ & $\begin{array}{l}\bar{\infty} \\
\tilde{N} \\
0\end{array}$ & 总 & $\stackrel{n}{\stackrel{n}{0}}$ & $\frac{\mathfrak{m}}{\stackrel{5}{0}}$ & $\begin{array}{l}\overline{\tilde{N}} \\
\text { on }\end{array}$ & $\stackrel{\infty}{\stackrel{\infty}{\circ}}$ & $\begin{array}{l}\text { ô } \\
\text { o. }\end{array}$ & 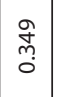 & $\begin{array}{l}\text { Do } \\
\stackrel{0}{0} \\
0\end{array}$ & $\underset{\widetilde{N}}{\stackrel{\sim}{0}}$ & $\frac{2}{5}$ & $\begin{array}{l}\stackrel{0}{0} \\
\stackrel{0}{0}\end{array}$ \\
\hline $\bar{i}$ & 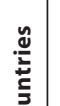 & $\underset{\stackrel{m}{\sim}}{\stackrel{\sim}{0}}$ & $\begin{array}{l}\overline{0} \\
\stackrel{0}{0} \\
0\end{array}$ & $\begin{array}{l}\overline{0} \\
\stackrel{n}{\circ} \\
0\end{array}$ & $\begin{array}{l}\tilde{0} \\
\dot{0} \\
0 \\
0\end{array}$ & $\begin{array}{l}\hat{n} \\
\hat{0} \\
0 \\
0\end{array}$ & 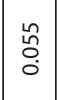 & $\begin{array}{l}\bar{\alpha} \\
\hat{0} \\
0\end{array}$ & $\begin{array}{l}\stackrel{i}{\stackrel{T}{*}} \\
\stackrel{\circ}{0}\end{array}$ & 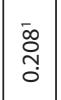 & 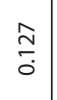 & 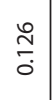 & $\begin{array}{l}0 \\
\stackrel{0}{0} \\
0\end{array}$ & $\begin{array}{l}\bar{n} \\
\stackrel{1}{\circ} \\
\end{array}$ & $\begin{array}{l}\bar{\sigma} \\
\stackrel{\infty}{\infty} \\
\dot{\sigma}\end{array}$ & $\begin{array}{l}\stackrel{a}{0} \\
\stackrel{0}{0}\end{array}$ & 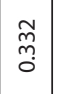 & $\begin{array}{l}\bar{\infty} \\
\stackrel{0}{0} \\
0\end{array}$ & $\frac{\circ}{\circ}$ & $\stackrel{\frac{\pi}{2}}{0}$ & $\begin{array}{l}\text { o } \\
\stackrel{0}{0}\end{array}$ \\
\hline$\frac{0}{i}$ & 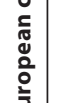 & $\frac{7}{0}$ & $\begin{array}{l}\bar{\infty} \\
\infty \\
\overline{0}\end{array}$ & $\begin{array}{l}\stackrel{\bar{n}}{h} \\
\frac{0}{0}\end{array}$ & $\begin{array}{l}\bar{\sigma} \\
\frac{0}{0}\end{array}$ & $\underset{0}{\stackrel{2}{\check{0}}}$ & $\begin{array}{l}\text { Iี } \\
\text { ơ }\end{array}$ & $\begin{array}{l}\bar{n} \\
\hat{0} \\
0\end{array}$ & $\frac{\bar{E}}{\overline{0}}$ & 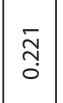 & $\frac{\Phi}{\infty}$ & $\begin{array}{l}\text { mo } \\
\text { ò }\end{array}$ & $\begin{array}{l}\bar{\infty} \\
\overline{0} \\
\overline{0}\end{array}$ & $\frac{\bar{\alpha}}{\infty}$ & $\begin{array}{l}\overline{\tilde{\nu}} \\
\stackrel{\infty}{\circ} \\
\bar{\sigma}\end{array}$ & $\stackrel{\infty}{\check{0}}$ & 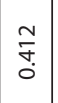 & $\begin{array}{l}\hat{\tilde{o}} \\
\stackrel{0}{0} \\
\text { Oे }\end{array}$ & 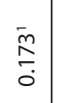 & $\frac{\pi}{5}$ & $\begin{array}{l}\text { o } \\
\text { o } \\
0\end{array}$ \\
\hline ஓे & 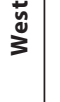 & $\stackrel{0}{\frac{0}{0}}$ & $\frac{\bar{c}}{\stackrel{0}{0}}$ & $\frac{\bar{\infty}}{\bar{\infty}}$ & $\begin{array}{l}m \\
\ddot{0} \\
0\end{array}$ & $\stackrel{\infty}{\infty}$ & $\stackrel{\frac{5}{n}}{0}$ & $\begin{array}{l}0 \\
\stackrel{0}{0} \\
0 \\
0\end{array}$ & $\frac{\overline{0}}{\circ}$ & \begin{tabular}{|l}
$\overline{\tilde{O}}$ \\
Oె
\end{tabular} & 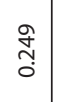 & $\begin{array}{l}\overline{\check{O}} \\
\frac{0}{\circ}\end{array}$ & $\mid \begin{array}{c}\infty \\
\stackrel{\infty}{0} \\
\overline{0}\end{array}$ & $\frac{\bar{\alpha}}{\circ}$ & 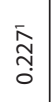 & $\stackrel{n}{\circ}$ & \begin{tabular}{l}
$\stackrel{\omega}{\infty}$ \\
\hdashline \\
0 \\
0
\end{tabular} & $\begin{array}{l}\text { స్ర } \\
\stackrel{0}{0}\end{array}$ & 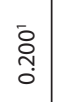 & $\begin{array}{l}n \\
\stackrel{n}{0}\end{array}$ & 命 \\
\hline$\stackrel{\infty}{\stackrel{0}{*}}$ & & $\frac{\overline{0}}{\circ}$ & $\begin{array}{l}\bar{\sigma} \\
\stackrel{0}{0} \\
\end{array}$ & $\begin{array}{l}\overline{\hat{\sigma}} \\
\frac{0}{0}\end{array}$ & $\begin{array}{l}1 \\
\text { ò } \\
\text {. }\end{array}$ & $\begin{array}{l}\stackrel{0}{0} \\
\text { o. }\end{array}$ & $\begin{array}{l}\bar{\circ} \\
\text { o. } \\
\text { o. }\end{array}$ & $\begin{array}{l}\bar{n} \\
o \\
o \\
0\end{array}$ & $\begin{array}{l}\text { б. } \\
\text { ơ }\end{array}$ & 1 & $\begin{array}{l}\text { बे } \\
\text { ○े }\end{array}$ & $\frac{8}{\circ}$ & $\frac{m}{m}$ & $\frac{\pi}{0}$ & $\stackrel{0}{\underset{丶}{0}}$ & $\begin{array}{l}\infty \\
o \\
0 \\
0\end{array}$ & ஷ̊. & $\begin{array}{l}\frac{m}{n} \\
0 \\
0\end{array}$ & ठัญ & $\frac{\hat{0}}{0}$ & oิ \\
\hline ¿্் & & $\frac{\stackrel{\sim}{\Im}}{0}$ & $\begin{array}{l}\bar{\infty} \\
\stackrel{0}{0}\end{array}$ & $\frac{\overline{0}}{\stackrel{0}{0}}$ & \begin{tabular}{l}
$\stackrel{0}{\circ}$ \\
\hdashline
\end{tabular} & $\stackrel{0}{\stackrel{0}{0}}$ & $\begin{array}{l}\bar{\infty} \\
\stackrel{0}{0}\end{array}$ & $\underset{\bar{\sigma}}{\bar{\sigma}}$ & $\begin{array}{l}\stackrel{0}{0} \\
\stackrel{0}{0}\end{array}$ & $\begin{array}{l}\infty \\
\stackrel{\infty}{0}\end{array}$ & 芯 & $\stackrel{\substack{n \\
0}}{\circ}$ & $\begin{array}{l}\overline{8} \\
\stackrel{0}{\circ}\end{array}$ & $\stackrel{?}{\stackrel{2}{0}}$ & $\underset{0}{\stackrel{t}{\circ}}$ & $\stackrel{\hat{m}}{\frac{0}{0}}$ & $\stackrel{n}{\frac{n}{7}}$ & 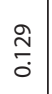 & 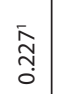 & $\begin{array}{l}\infty \\
\stackrel{\infty}{0} \\
\end{array}$ & $\begin{array}{l}\infty \\
\stackrel{0}{0} \\
0\end{array}$ \\
\hline ঃ & & $\frac{\bar{\sigma}}{0}$ & 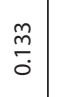 & $\frac{\bar{y}}{\overline{0}}$ & $\begin{array}{l}\frac{1 n}{\circ} \\
\frac{0}{0}\end{array}$ & 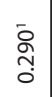 & $\begin{array}{l}\hat{\widehat{a}} \\
\text { o } \\
\text { o }\end{array}$ & $\begin{array}{l}\overline{0} \\
o \\
0 \\
0\end{array}$ & চ̊. & 1 & 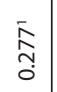 & 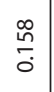 & $\frac{\bar{\sigma}}{0}$ & $\stackrel{\infty}{\underset{0}{0}}$ & $\frac{\bar{m}}{\frac{\bar{o}}{o}}$ & $\begin{array}{l}\text { oे } \\
\text { Oें }\end{array}$ & 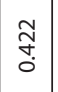 & $\stackrel{\searrow}{\circ}$ & $\begin{array}{l}\overline{0} \\
\stackrel{n}{n} \\
0\end{array}$ & $\begin{array}{l}\underset{N}{0} \\
0\end{array}$ & $\begin{array}{l}8 \\
8 \\
0\end{array}$ \\
\hline ڤ̊̊ & & $\frac{8}{\circ}$ & $\frac{\bar{i}}{\tilde{n}}$ & $\begin{array}{l}\frac{i n}{\stackrel{n}{n}} \\
\frac{0}{0}\end{array}$ & $\begin{array}{l}\stackrel{\llcorner}{0} \\
\stackrel{0}{0}\end{array}$ & $\stackrel{\infty}{\check{0}}$ & $\frac{\overline{0}}{\circ}$ & $\begin{array}{l}\bar{\infty} \\
\hat{O} \\
0 \\
0\end{array}$ & $\begin{array}{l}\overline{\bar{o}} \\
\bar{\infty} \\
0 \\
0\end{array}$ & 1 & $\underset{\substack{\text { ja } \\
\text { d }}}{ }$ & ठ্ণ & $\frac{\pi}{\sigma}$ & $\frac{\bar{\alpha}}{\bar{\infty}}$ & $\begin{array}{l}\overline{\check{o}} \\
\text { ơ }\end{array}$ & $\bar{\varnothing}$ & $\begin{array}{l}\mathbb{J} \\
\stackrel{m}{o}\end{array}$ & $\frac{\circ}{\frac{0}{0}}$ & $\frac{\bar{j}}{\frac{5}{0}}$ & $\stackrel{\infty}{\stackrel{\infty}{\circ}}$ & $\hat{o}_{0}^{n}$ \\
\hline ষ্ণ & & $\frac{\infty}{\stackrel{\infty}{\circ}}$ & $\begin{array}{l}\bar{o} \\
\stackrel{0}{0} \\
\end{array}$ & $\stackrel{\stackrel{t}{m}}{\stackrel{0}{0}}$ & ồ & $\stackrel{\nwarrow}{\stackrel{1}{0}}$ & $\frac{\circ}{\frac{1}{0}}$ & $\begin{array}{l}\bar{d} \\
\frac{0}{0}\end{array}$ & $\frac{\bar{i}}{\frac{m}{o}}$ & 1 & $\underset{\text { ָ̦ }}{\text { ஸे }}$ & $\begin{array}{l}\bar{\circ} \\
\stackrel{\circ}{\circ} \\
\end{array}$ & $\begin{array}{l}\stackrel{n}{\leftarrow} \\
\stackrel{0}{0}\end{array}$ & $\begin{array}{l}\bar{\infty} \\
\frac{\infty}{\sigma} \\
\end{array}$ & $\frac{n}{\circ}$ & 1 & 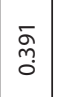 & 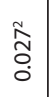 & $\frac{\bar{y}}{\stackrel{y}{0}}$ & $\frac{n}{0}$ & $\begin{array}{l}\hat{\partial} \\
\hat{O} \\
0\end{array}$ \\
\hline & & を & 山् & 山् & 吕 & $\overrightarrow{\text { 山 }}$ & นั & $\overline{\mathbf{u}}$ & 稆 & I & $\underline{\underline{ }}$ & $\underline{\simeq}$ & $\boldsymbol{E}$ & ב & $\vec{z}$ & 울 & เ & 嵓 & 号 & $\frac{\Sigma}{\Sigma}$ & 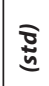 \\
\hline
\end{tabular}




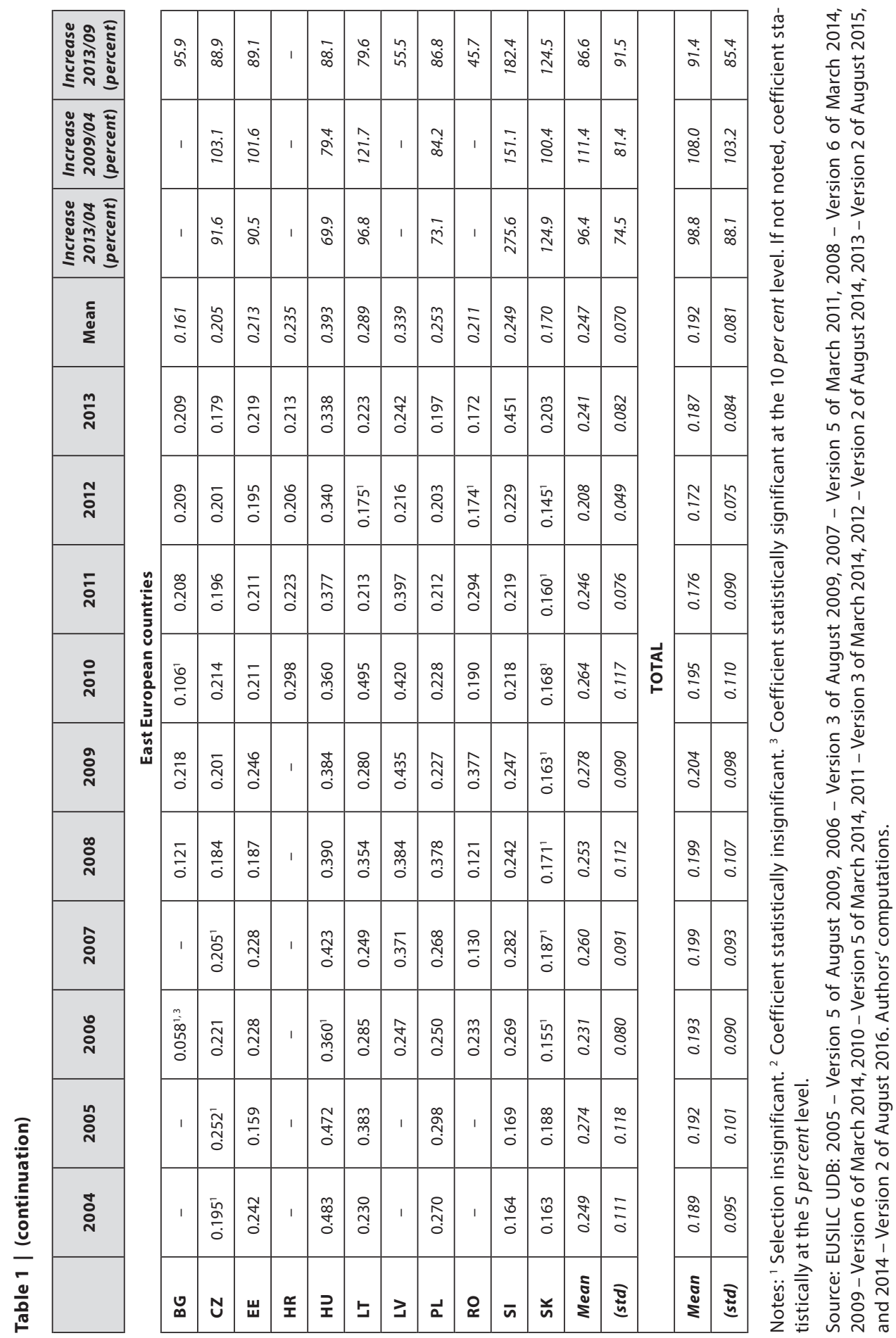




\section{A Cross-Europe Comparison in the 2000s: the Macro-Level}

In this section, we aim to test the hypothesis that an increase in the supply of educated labour reduces its marginal product and thus the returns to education. In recent decades, European countries have experienced a boom in tertiary education. According to the ILO database, we can estimate that on average this percentage in Western countries was about 20 per cent in the beginning of the 1990s and it reached 32 per cent in 2012. In transition countries, the percentage was about 14 per cent in the 1990s and it reached 27 per cent in 2012. Evidently, the number of workers with tertiary education grew faster in transition countries and despite the continuing rise in the West the gap between the two groups of countries decreased.

Figure 1 | Returns to Tertiary Education by the Share of Tertiary Educated in Working Age Population (regression coefficients in Heckman model)

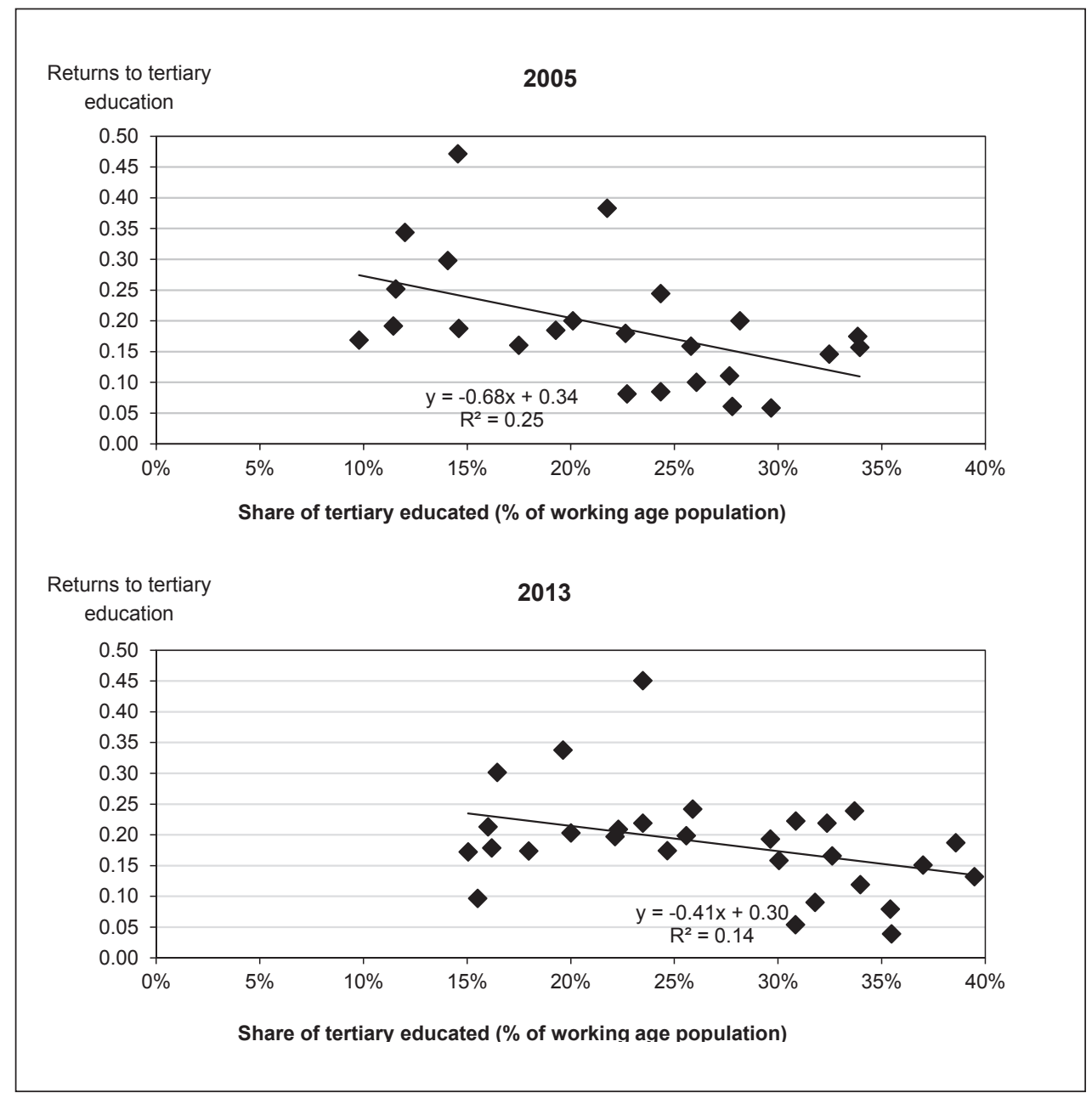


Figure 1 | (continuation)

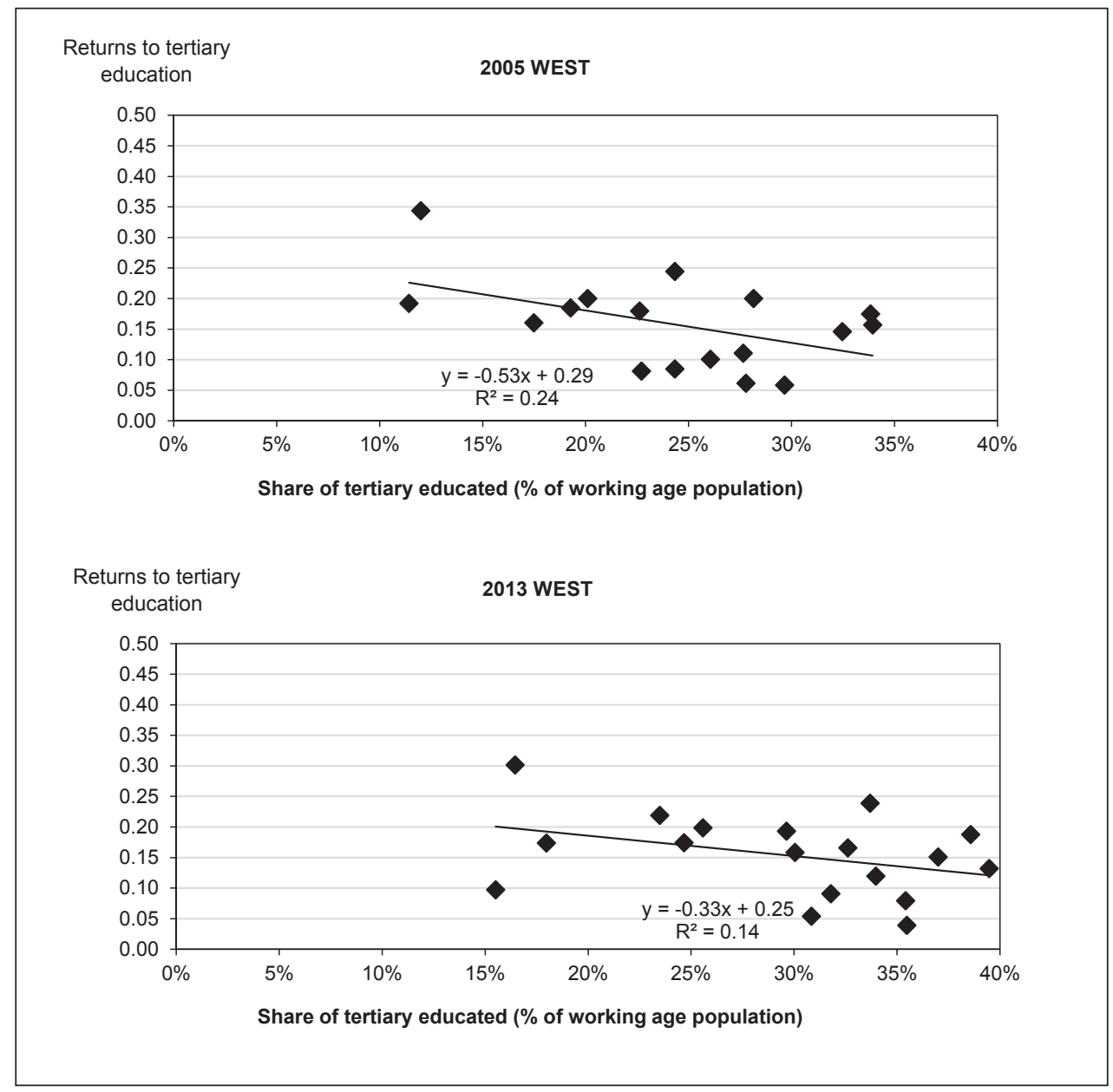

To begin, we examine the simple relationship between returns to tertiary education estimated with the Heckman model in the previous section and the percentage of people with tertiary education among the working-age population (Figure 1). When comparing the two time observations, we can first see that, in general, the returns to education are indeed lower in countries with a higher share of educated workers. However, this relationship only applies in Western countries, while there is no such relationship in transition countries. Second, the relationship became looser over time in the West, while rather the opposite occurred in the East. ${ }^{2}$ This suggests that the Eastern labour markets are far from saturated with tertiary-educated workers.

2 Bulgaria, Croatia, and Romania are not included in the 2005 sample. 
Figure 1 | (continuation)

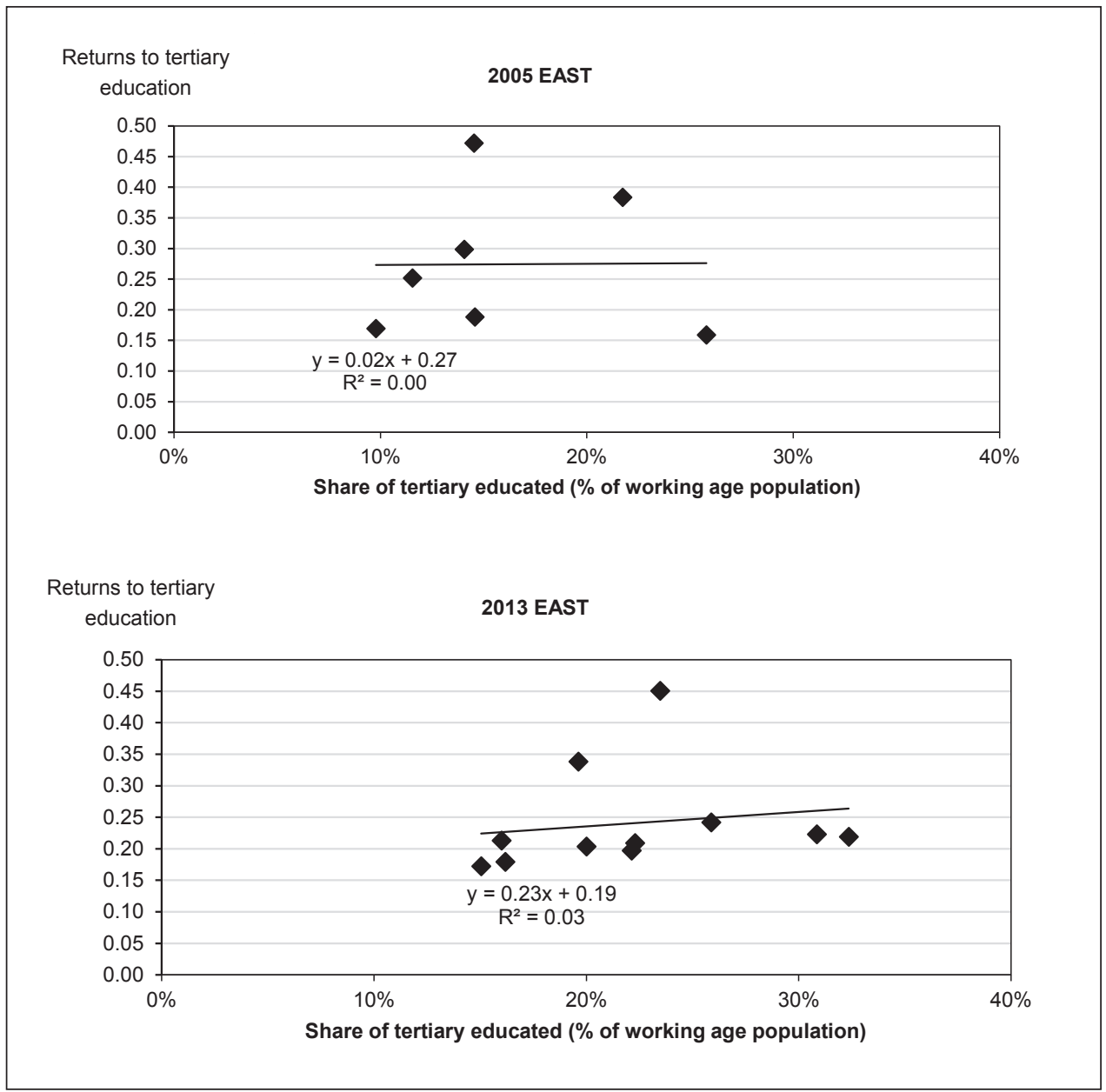

Notes: For 2005, returns to tertiary education stem from EU-SILC 2006 and share of tertiary educated from EU-SILC 2005; for 2011, returns to tertiary education stem from EU-SILC 2012 and share of tertiary educated from EU-SILC 2011.

Source: EUSILC UDB: 2005 - Version 5 of August 2009, 2006 - Version 3 of August 2009, 2013 - Version 2 of August 2015, and 2014 - Version 2 of August 2016. Authors' computations.

\subsection{Methodology and data}

The more detailed analysis in this section uses panel regression models with countries as the unit of observation. For all the models presented below, the Hausman test (Greene, 2008) indicates that the random-effects model is more suitable than the fixed-effects model. Furthermore, the Breusch-Pagan (Breusch and Pagan, 1980) LM test confirmed 
that the random-effects panel model is more fitting than the simple OLS regression for all the models. Robust standard errors are applied to avoid heteroscedasticity. The random effects model is

$$
Y_{i t}=\alpha+\beta X_{i t}+u_{i t}+\varepsilon_{i t},
$$

Where $u_{i t}$ is the between-country error and $\varepsilon_{i t}$ is the within-country error term. The dependent variable is return to tertiary education for each country $i$ and for year $t$ obtained from the Heckman selection models based on the cross-sectional individual data described in the previous section. Vector $X_{i t}$ includes explanatory variables. The variable of interest is the share of individuals with tertiary education in the working-age population (16-65), as obtained from EU-SILC. As noted earlier, the income reference period in EU-SILC is the previous calendar year, while education is related to the time of the survey. Hence, each two subsequent waves are combined in order to determine the returns to tertiary education and the share of tertiary-educated people in time $t$. The panel data thus cover nine years, with $t$ ranging from 2005 to 2013 .

The set of control variables includes the following country-level indicators:

- Job vacancy rate (the number of job vacancies as a share of the number of occupied posts plus the number of job vacancies, Eurostat). Job vacancies serve as a measure of labour demand. An optimal variable for our purposes would be the ratio of vacancies for people with tertiary and secondary education, but such a division is not available in the Eurostat database. In most countries, the majority of employed people have secondary as opposed to tertiary education. In the majority of Eastern European countries this difference is as much as two-fold. Therefore, it can be expected that if labour demand increases, the demand for secondary-educated people will rise faster than the demand for tertiary-educated, the wage gap between tertiary- and secondaryeducated decreases, which would result in a negative impact on returns to tertiary education.

- Life expectancy at birth (Eurostat). Income inequality is negatively correlated with life expectancy (see among others Mayrhofer and Schmitz, 2014; seminal study by Preston, 1975). Longevity is a concave function of income on an individual level and, consequently, a more egalitarian income distribution leads to a higher life expectancy. In this sense, life expectancy might serve as a proxy for an overall tendency for a more egalitarian income distribution in a country and lower wage differentials, and thus, a negative impact on returns to tertiary education can be expected.

- Employment in services (as a percentage of total employment, Eurostat). Based on the empirics compared to manufacturing not only do services attract more tertiaryeducated people, but also the wages (in that sector) are higher (a positive gap between the average wage in services and manufacturing adjusted for skill composition has been demonstrated, for example, by Genre et al., 2005). A positive effect might therefore be expected.

- Implicit tax rate on labour (ratio of direct taxes, indirect taxes and compulsory actual social contributions paid by employers and employees to employed labour income, 
Eurostat). ${ }^{3}$ The effect of the tax rate is unclear and it mainly depends on the progressivity of the tax system (see among others Mendolicchio and Rhein, 2014).

Furthermore, several other country-level characteristics were tested: GDP - empirics show that returns to education are larger in poor countries, and so a negative impact on returns to education was expected (Banerjee and Duflo, 2005, p. 488); R\&D expenditure - the growing productivity of educated labour due to technological changes increases the demand for it (Mincer, 1991) and therefore the returns to education should grow; and trade union density -the empirical results are rather ambiguous, but several explanations for the "flattering" hypothesis can be found (Ashley, 1999) according to which the effect of education on wages for union members is lower than for non-union members, and, therefore, a negative impact was expected. None of them was significant in any model specification.

\subsection{Results of the analysis}

Table 2 shows the results of a basic panel regression with only the variable of our main interest and the regional dummies included. The expected negative impact of the share of tertiary-educated population on returns to education was confirmed for Europe as a whole (Model 1). ${ }^{4}$ After controlling for the share of tertiary education, the difference in returns to education between the Eastern and Western of Europe suggested by the results in the previous section are confirmed: A dummy for Eastern Europe indicates higher returns to tertiary education in this region (Model 2). The Chow test (Chow, 1960) confirmed that the Model coefficients for Eastern and Western Europe are significantly different. As Models (4) and (6) show, the negative impact of the share of tertiary education decreases returns to tertiary education only in Western Europe, while there is no significant effect in Eastern Europe. ${ }^{5}$

In order to capture the unmeasured effects of, among others, welfare system or culture within Europe, we added five dummies for various European regions: the Visegrád Four, the Baltics, the rest of Eastern Europe, Scandinavia and Southern Europe (with the rest of Western Europe for reference). Except for the Baltics (significantly positive effect) and

3 The latest data available are for 2012; as a result, the panel regression with control variables has to be shortened to eight years.

4 The causality between returns to tertiary education and the share of tertiary-educated population might be questioned. Granger's predictive causality test was performed in order to indicate that the share of tertiary-educated population Granger-causes returns to tertiary education and not vice versa. The test (including one and two lags) rejected the null hypothesis of no Granger causality, while the same cannot be said about the opposite direction. Moreover, an IV panel regression, where the share of tertiary-educated population was instrumented by its (up to three) lags, was run to further support the idea that the direction of the causality in the applied regression models is correct. The results were the same regarding the significance and the sign and the coefficients were even higher.

5 The Wald test indicated that the models for Eastern Europe are not appropriate; see Columns (4) and (5) in Table 2. 
Scandinavia (significantly negative effect), these coefficients did not show a significant impact on returns to education either in the model for Europe as a whole or in the models for Eastern and Western Europe.

Table 2 | Returns to Education - Panel Regression, Random Effects, 2005-2013

\begin{tabular}{|c|c|c|c|c|c|c|c|}
\hline & $\begin{array}{c}\text { (Model 1) } \\
\text { Europe }\end{array}$ & $\begin{array}{c}\text { (Model 2) } \\
\text { Europe }\end{array}$ & $\begin{array}{l}\text { (Model 3) } \\
\text { Europe }\end{array}$ & $\begin{array}{c}\text { (Model 4) } \\
\text { East }\end{array}$ & $\begin{array}{c}\text { (Model 5) } \\
\text { East }\end{array}$ & $\begin{array}{c}\text { (Model 6) } \\
\text { West }\end{array}$ & $\begin{array}{c}\text { (Model 7) } \\
\text { West }\end{array}$ \\
\hline $\begin{array}{l}\text { Share of tertiary } \\
\text { educated }\end{array}$ & $-0.432 * * *$ & $-0.369^{* *}$ & $-0.384^{* *}$ & -0.031 & -0.174 & $-0.507^{* * *}$ & $-0.484^{* *}$ \\
\hline East & - & $0.063^{* *}$ & - & - & - & - & - \\
\hline V4 & - & - & 0.041 & - & 0.038 & - & - \\
\hline Baltic & - & - & $0.105^{* * *}$ & - & 0.082 & - & - \\
\hline Rest of EE & - & - & 0.003 & - & - & - & - \\
\hline Scandinavia & - & - & $-0.068^{* * *}$ & - & - & - & $-0.065^{* * *}$ \\
\hline South WE & - & - & -0.005 & - & - & - & -0.012 \\
\hline Constant & $0.293^{* * *}$ & $0.255^{* * *}$ & $0.275^{* * *}$ & $0.253^{* * *}$ & $0.244^{* * *}$ & $0.291^{* * *}$ & $0.302^{* * *}$ \\
\hline $\begin{array}{l}\text { Number } \\
\text { of observations }\end{array}$ & 245 & 245 & 245 & 87 & 87 & 158 & 158 \\
\hline Number of groups & 29 & 29 & 29 & 11 & 11 & 18 & 18 \\
\hline $\mathbf{R}^{2}$ within & 0.048 & 0.048 & 0.048 & 0.003 & 0.003 & 0.128 & 0.128 \\
\hline$R^{2}$ between & 0.216 & 0.339 & 0.515 & 0.033 & 0.196 & 0.201 & 0.354 \\
\hline $\mathbf{R}^{2}$ overall & 0.177 & 0.281 & 0.394 & 0.006 & 0.079 & 0.183 & 0.294 \\
\hline \multicolumn{8}{|c|}{ Tests p-values } \\
\hline Wald Chi ${ }^{2}$ & 0.007 & 0.000 & 0.000 & 0.912 & 0.225 & 0.008 & 0.000 \\
\hline Hausman & 0.499 & - & - & 0.473 & - & 0.932 & - \\
\hline B-P LM & 0.000 & 0.000 & 0.000 & 0.000 & 0.000 & 0.000 & 0.000 \\
\hline Chow & 0.000 & - & - & - & - & - & - \\
\hline
\end{tabular}

Notes: * statistically significant at the 10 per cent level, ** statistically significant at the 5 per cent level, *** statistically significant at the 1 per cent level.

Regions: East = all East European countries, V4 = Visegrád four $(C Z, H U, P L, S K)$, Baltic $=E E, L T$, LV, Rest of $E E=B G, H R, R O, S I$, Scandinavia $=D K, F I, I S, N O, S E$, South WE $=E L, E S, I T, P T$.

Source: EUSILC UDB: 2005 - Version 5 of August 2009, 2006 - Version 3 of August 2009, 2007 - Version 5 of March 2011, 2008 - Version 6 of March 2014, 2009 - Version 6 of March 2014, 2010 - Version 5 of March 2014, 2011 - Version 3 of March 2014, 2012 - Version 2 of August 2014, 2013 - Version 2 of August 2015, and 2014 - Version 2 of August 2016. Authors' computations. 
Table 3 | Returns to Education - Panel Regression, Random Effects, 2005-2012, Control Variables

\begin{tabular}{|c|c|c|c|c|c|c|c|}
\hline & $\begin{array}{l}\text { (Model 1) } \\
\text { Europe }\end{array}$ & $\begin{array}{l}\text { (Model 2) } \\
\text { Europe }\end{array}$ & $\begin{array}{l}\text { (Model 3) } \\
\text { Europe }\end{array}$ & $\begin{array}{c}\text { (Model 4) } \\
\text { East }\end{array}$ & $\begin{array}{c}\text { (Model 5) } \\
\text { East }\end{array}$ & $\begin{array}{l}\text { (Model 6) } \\
\text { West }\end{array}$ & $\begin{array}{c}\text { (Model 7) } \\
\text { West }\end{array}$ \\
\hline $\begin{array}{l}\text { Share } \\
\text { of tertiary } \\
\text { educated }\end{array}$ & $-0.495^{* *}$ & $-0.499 * *$ & $-0.532^{* *}$ & -0.373 & -0.425 & $-0.432^{* *}$ & $-0.429 *$ \\
\hline East & - & 0.006 & - & - & - & - & - \\
\hline V4 & - & - & 0.020 & - & -0.002 & - & - \\
\hline Baltic & - & - & 0.056 & - & 0.012 & - & - \\
\hline Rest of EE & - & - & -0.038 & - & - & - & - \\
\hline Scandinavia & - & - & -0.045 & - & - & - & -0.008 \\
\hline South WE & - & - & 0.010 & - & - & - & -0.049 \\
\hline $\begin{array}{l}\text { Job vacancy } \\
\text { rate }\end{array}$ & $-0.021^{*}$ & -0.020 & -0.018 & $-0.033^{* *}$ & $-0.032^{* *}$ & 0.006 & 0.005 \\
\hline Life expectancy & $-0.014^{* *}$ & $-0.013^{* *}$ & -0.010 & $-0.021^{* * *}$ & $-0.019 * *$ & -0.002 & 0.003 \\
\hline $\begin{array}{l}\text { Empl. } \\
\text { in services }\end{array}$ & 0.004 & 0.004 & 0.003 & $0.006^{* * *}$ & $0.006^{* * *}$ & -0.002 & -0.004 \\
\hline Tax rate & 0.003 & -0.003 & -0.003 & $0.007^{* *}$ & 0.006 & $-0.007^{* * *}$ & $-0.008^{* *}$ \\
\hline Constant & $1.307^{* * *}$ & $1.264^{* * *}$ & $1.004^{*}$ & $1.369^{* * *}$ & $1.293^{* *}$ & 0.817 & 0.591 \\
\hline $\begin{array}{l}\text { Number } \\
\text { of observations }\end{array}$ & 164 & 164 & 164 & 73 & 73 & 91 & 91 \\
\hline $\begin{array}{l}\text { Number } \\
\text { of groups }\end{array}$ & 25 & 25 & 25 & 11 & 11 & 14 & 14 \\
\hline$R^{2}$ within & 0.070 & 0.070 & 0.071 & 0.067 & 0.069 & 0.153 & 0.158 \\
\hline$R^{2}$ between & 0.534 & 0.531 & 0.640 & 0.698 & 0.689 & 0.697 & 0.724 \\
\hline $\mathbf{R}^{2}$ overall & 0.451 & 0.450 & 0.503 & 0.455 & 0.452 & 0.577 & 0.605 \\
\hline \multicolumn{8}{|c|}{ Tests $p$-values } \\
\hline Wald Chi ${ }^{2}$ & 0.000 & 0.001 & 0.000 & 0.000 & 0.000 & 0.000 & 0.000 \\
\hline Hausman & 0.854 & - & - & 0.437 & - & 0.601 & - \\
\hline B-P LM & 0.000 & 0.000 & 0.000 & 0.002 & 0.031 & 0.000 & 0.000 \\
\hline Chow & 0.000 & - & - & - & - & - & - \\
\hline
\end{tabular}

Notes: * statistically significant at the 10 per cent level, ** statistically significant at the 5 per cent level, *** statistically significant at the 1 per cent level. Four countries were excluded due to missing data (DK, IS, IT, CH).

Source: EU-SILC for returns to education and the share of tertiary educated population (see Table 2). For other source see the Section 4.1 Authors' computations. 
Table 3 shows the final results including the control variables described in previous subsection. Only variables statistically significant at least at the 10 per cent level in at least one region were finally included into the analysis. The negative impact of the share of tertiary education remains significant for Europe as a whole. As opposed to the basic model in Table 2, the dummy variable for Eastern Europe is not significant. This means that after controlling for national relevant variables, the gap in returns to tertiary education is no longer remarkable. The Chow test indicates that it is appropriate to run the model separately for Eastern and Western Europe. Moreover, the Chow predictive tests, which test a subgroup versus the total sample, suggest that the West European subsample should be separated while the East European subsample should not. ${ }^{6}$ The models for Eastern Europe therefore seem unstable.

The results in the Eastern and Western of Europe differ substantially. The negative relationship between the share of tertiary-educated people and returns to education was confirmed only in Western Europe. In the East, the share of tertiary-educated people has no effect on returns to tertiary education. On the contrary, the job vacancy rate has a significant negative impact on returns to tertiary education in Eastern Europe and no effect in Western Europe. It appears that in the West, the labour market reacts to labour supply, while labour demand plays a more important role in the East.

Regarding the other indicators, the indicator life of expectancy showed a significant negative effect in the East. Employment in services increases returns to tertiary education only in the East. The tax rate showed a significant effect in both the West and the East, but while the effect on returns to tertiary education was positive in the East, it was negative in the West. The higher the taxes on labour in Western Europe are, the lower the difference in wages is for workers with tertiary and secondary education. Thus, while the tax system has an equalising effect on educational wage differences in Western Europe, the effect is quite the opposite in Eastern Europe.

In both groups of countries, the explained within-country variance in returns to tertiary education is considerably smaller than the explained between-country variance. Consequently, the variables included in the models capture differences among countries relatively well, while they fail to explain changes over time within countries in the period under observation. The difference between the East and the West of Europe has remained about the same since the mid-2000s, unlike the increasing similarity of the two regions regarding returns to education and the eventual superiority of the Eastern European countries occurred in the 1990 s.

6 The Chow predictive test is a variation of Chow test. When Western Europe represents a subsample of Europe, we can reject the null hypothesis of parameter constancy, while when Eastern Europe is tested versus the whole Europe, we cannot. The statistics are: $F=\frac{\frac{S S R_{T}-S S R_{1}}{n_{2}}}{\frac{S S R_{1}}{n_{1}-k}} \sim F\left(n_{2}, n_{1}-k\right)$ 


\section{Conclusion}

In Western countries the empirics of returns to education have for decades been addressed in numerous studies. Most of them show quite different levels of returns to education and fail to identify any uniform trend for the 1980s and 1990s. Instead, they present a rather mixed picture of small increases or decreases and often almost a stability of returns to education. The evidence thus does not clearly indicate how and owing to what factors (and, after all, in conformity with which theories) returns to education changed in advanced economies over time.

For transition countries there are far fewer studies. Nevertheless, since 1990, with the advent of new regimes across the region and the availability of large statistical surveys, research in this area has been growing. Most studies concur that there has been a uniform upward trend in rewards to higher education. As was expected, in the place where education was deliberately degraded during the period of "working-class rule", there occurred a change as education started to be valued under the market system and democratic regime. This change was often substantial and fast. For instance, returns to education in the Czech Republic for one year of schooling doubled between 1988 and 1996.

Since the mid-2000s, the EU-SILC surveys have served as a unique database for Europe. The data show some volatility of returns to higher education within a narrow range. We can cautiously point to data from 2009 as a kind of turning point, when returns to education that had been rising or stable to that time started to decrease slightly. Changes over time have always been much smaller than the differences between countries, which have changed little. Unlike the small changes over time, the range of cross-country differences is quite wide.

As regards the factors behind the different returns to education across countries, there are substantial differences between the Eastern and the Western of Europe. The expected negative relationship between the proportion of tertiary-educated in the active population and returns to education was confirmed only in the West. By contrast, the job vacancy rate has a significant negative impact on returns to tertiary education only in the East. This result suggests that in Western Europe the labour market reacts to labour supply, while labour demand plays a more important role in Eastern Europe.

In both groups of countries, the explained within-country variance in returns to tertiary education is considerably smaller than the explained between-country variance of returns. The variables included in the models capture the differences between countries relatively well, but they fail to explain changes over time within countries. The difference between the East and the West has remained about the same since the mid-2000s, in contrast to the striking trend of decreasing differences during the 1990s due to the rapid increase of returns to education in transition countries. Returns to tertiary education are thus still considerably higher in East Europe than in Western countries. 


\section{References}

Ashley, T. (1999). Union Membership Effects on Black-White Differences in Returns to Education. Review of Black Political Economy, 27(2), 13-32, https://doi.org/10.1007/s12114-999-1030-3

Banerjee, A. V., Duflo, E. (2005). Growth Theory through the Lens of Development Economics. In Handbook of Economic Growth, 1A, pp. 473-552, edited by Aghion, P., Durlauf, S. N. Amsterdam: North-Holland.

Barth, E., Lucifora, C. (2006). Wage Dispersion, Markets and Institutions: the Effects of the Boom in Education on the Wage Structure. IZA. Discussion Papers No. 2181, 1-42.

Becker, G. S. (1975). Human Capital and the Personal Distribution of Income: An Analytical Approach. In Becker, G. S. Human Capital: A Theoretical and Empirical Analysis, with Special Reference to Education. 2nd Edition. New York: National Bureau of Economic Research.

Björklund, A. (2000). Education Policy and Returns to Education. Swedish Economic Policy Review, 7(1), 71-105.

Breusch, T. S., Pagan, A. R. (1980). The Lagrange Multiplier Test and its Applications to Model Specification in Econometrics. Review of Economic Studies, 47(1), 239-253, https://doi.org/10.2307/2297111

Chow, G. C. (1960). Tests of Equality between Sets of Coefficients in Two Linear Regressions. Econometrica, 28(3), 591-605, https://doi.org/10.2307/1910133

Dolton, P., Asplund, R., Barth, E. (2009). Education and Inequality across Europe. Cheltenham, UK and Northampton, MA: Edward Elgar. ISBN 9781847205889.

EDWIN (2006). Education and Wage Inequality in Europe - final report. Available at: http://www.etla.fi/edwin/HPSE-CT-2002-00108_EDWIN_Final_report_16012006.pdf

Fersterer, J., Winter-Ebmer, R. (2003). Are Austrian Returns to Education Falling over Time? Labour Economics, 10(1), 73-89, https://doi.org/10.1016/s0927-5371(02)00105-7

Filer, R. K., Jurajda, Š., Plánovský, J. (1999). Education and Wages in the Czech and Slovak Republics during Transition. Labour Economics, 6(4), 581-593, https://doi.org/10.1016/ s0927-5371(99)00032-9

Flanagan, R. (1998). Were Communists Good Human Capitalists? The Case of the Czech Republic. Labour Economics, 5(3), 295-312, https://doi.org/10.1016/s0927-5371(97)00029-8

Fleisher, B. M., Sabirianova, K., Wang, X. (2005). Returns to Skills and the Speed of Reforms: Evidence from Central and Eastern Europe, China, and Russia. Journal of Comparative Economics, 33(2), 351-370, https://doi.org/10.1016/j.jce.2005.03.003

Genre, V., Momferatou, D., Mourre, G. (2005). Wage Diversity in the Euro Area. An Overview of Labour Costs Differentials across Industries. ECB. Occasional Paper, 24, 1-47.

Greene, W. H. (2008). Econometric Analysis, 6th edition. Upper Saddle River, NJ: Prentice Hall.

Harmon, C., Walker, I., Westergård-Nielsen, N. (2001). Education and Earnings in Europe: A Cross Country Analysis of the Returns to Education. Cheltenham: Edward Elgar. ISBN 9781840645309.

Heckman, J. J. (1979). Sample Selection Bias as a Specification Error. Econometrica, 47(1), 153-161, https://doi.org/10.2307/1912352

Jurajda, Š. (2003). Gender Wage Gap and Segregation in Enterprises and the Public Sector in Late Transition Countries. Journal of Comparative Economics, 31(2), 199-222, https://doi.org/10.1016/s0147-5967(03)00040-4 
Kertesi, G., Köllő, J. (1999). Economic Transformation and the Return to Human Capital. The Case of Hungary, 1986-1996. Budapest Working Papers on the Labour Market 6.

Mayrhofer, T., Schmitz, H. (2014). Testing the Relationship between Income Inequality and Life Expectancy: A Simple Correction for the Aggregation Effect when Using Aggregated Data. Journal of Population Economics, 27(3), 841-856, https://doi.org/10.1007/s00148-013-0483-7

Mendolicchio, C., Rhein, T. (2014). The Gender Gap of Returns on Education across West European Countries. International Journal of Manpower, 35(3), 219-249, https://doi.org/10.1108/ ijm-02-2013-0026

Mincer, J. (1974). Schooling, Experience and Earnings. New York: Columbia University Press.

Mincer, J. (1991). Human Capital, Technology, and the Wage Structure: What do Time Series Show? NBER. Working Paper No. 3581, 1-54, https://doi.org/10.3386/w3581

Moffitt, R. (2007). Estimating Marginal Returns to Higher Education in the UK. NBER. Working Paper No. 13534, 1-39, https://doi.org/10.3386/w13534

Munich, D., Švejnar, J., Terrell, K. (2005). Returns to Human Capital under the Communist Wage Grid and during the Transition to a Market Economy. Review of Economics and Statistics, 87(1), 100-123, https://doi.org/10.1162/0034653053327559

Mysíková, M., Večerník, J. (2015). Returns to Education in Transition and Advanced European Countries: The Role of an Expansion of Higher Education. XXIV Jornadas de la Asociación de Economía de la Educación. Investigaciones de economía de la educación 10, pp. 865-886. Available at: http://repec.economicsofeducation.com/2015madrid/madrid2015.pdf

Preston, S. H. (1975). The Changing Relation between Mortality and Level of Economic Development. Population Studies, 29(2), 231-248, https://doi.org/10.1080/00324728.1975.1 0410201

Psacharopoulos, G. (1989). Time Trends of the Returns to Education: Cross-national Evidence. Economics of Education Review, 8(3), 225-231, https://doi.org/10.1016/0272-7757 (82)90002-4

Psacharopoulos, G., Patrinos, H. A. (2004). Returns to Investment in Education: A Further Update. Education Economics, 12(2), 111-134, https://doi.org/10.1080/0964529042000239140

PURE (2001). The TSER-financed Project on Public Funding and Private Returns to Education - Final Report, Available at: http://cordis.europa.eu/documents/ documentlibrary/70781481EN6.pdf

Rutkowski, J. (2001). Earnings Inequality in Transition Economies of Central Europe. Trends and Patterns during the 1990s. SP Discussion Papers No. 0117, 1-54.

Stanovnik, T. (1997). The Returns to Education in Slovenia. Economics of Education Review, 16(4), 443-449, https://doi.org/10.1016/s0272-7757(97)00006-x

Thélot, C., Selz, M. (2004). The Returns to Education and Experience: Trends in France over the Last Thirty-Five Years. Population, 59(1), 9-47, https://doi.org/10.2307/3654927

Večerník, J. (2009). Czech Society in the 2000s: A Report on Socio-Economic Policies and Structures. Praha: Academia. ISBN 97880201750-5.

Večerník, J. (2013). The Changing role of Education in the Distribution of Earnings and Household Income. The Czech Republic, 1988-2009. Economics of Transition, 21(1), 111-133, https://doi.org/10.1111/ecot.12002 